\title{
Temperature prediction of PV/T component based on RBF neural
}

\section{network}

\author{
Jiyong $\mathrm{Li}^{1,2, \mathrm{a}}$, Zhendong Zhao ${ }^{1,2, \mathrm{~b}}$,Junpei $\mathrm{Nan}^{1,2, \mathrm{c}}$,Yisheng $\mathrm{Li}^{1,2, \mathrm{~d}}$, \\ Yunfeng Tang ${ }^{1,2, \mathrm{e}}$ \\ 1 Guangxi University, Department of Electrical Engineering, Nanning 530004, Guangxi, \\ Peoples R China \\ 2 Guangxi Key Laboratory of Power System Optimization and Energy Technology(Guangxi \\ University), Nanning, Guangxi 530004, China \\ aji_yong_li@163.com, \\ bmr.zzd@163.com, ,240117926@qq.com,,539968384@qq.com, ${ }^{\mathrm{e}} 1584483139 @ q q . c o m$
}

Key words: PV/T; Optimal control; Temperature prediction; RBF neural network

Abstract: In the comprehensive utilization of solar photovoltaic-thermal hybrid (PV/T) system research, $\mathrm{PV} / \mathrm{T}$ component temperature control is very important to improve the efficiency and thermal efficiency. For PV/T system is a big inertia system, temperature control effect needs a period of time. So accurately predicting PV/T component temperature in advance is advantageous to the PV/T system optimization control. In this paper, based on RBF neural network, the sunny weather temperature prediction model is established, which can forecast the component temperature after 15 minutes. By comparing measured data with the predicted data, the model has a high accuracy in prediction, which can provide technical support for the follow-up PV/T system research.

\section{Introduction}

The conversion efficiency of photovoltaic power generation (PV) system is only about $10 \%$ to $20 \%$, part of the solar radiation reflection back to the outside, all the other into heat. Sunny weather, PV module maximum temperature is up to $80{ }^{\circ} \mathrm{C}$ or so in Guangxi Nanning. Solar radiation and PV component temperature have a great impact on the photovoltaic array power generation efficiency. The research result shows that [1,2], the crystalline silicon solar cell module junction temperature exceeds $25{ }^{\circ} \mathrm{C}$, the power will be reduced by about $0.5 \%$ per degree Celsius.

In order to reduce the temperature of PV module, improve the efficiency of power generation and recover the heat generated by PV module, the PV/T system, which integrates solar power and heat utilization, is attracting more and more attention. Fig. 1 shows the flat-type monocrystalline silicon PV/T component structure diagram, cold water through the copper pipe to take away the heat, then repeated cycle. Fig. 2 shows the PV/T system diagram, through the controller to adjust the pump speed, and then adjust the flow rate. 


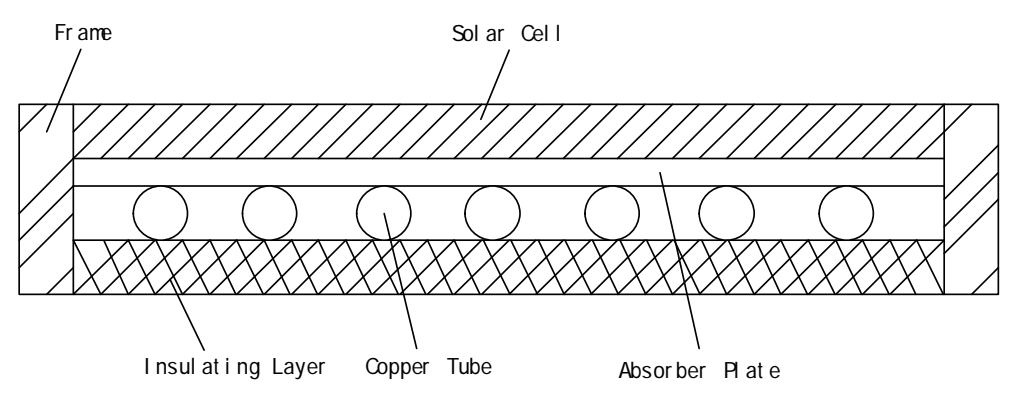

Fig. 1 PV / T component structure

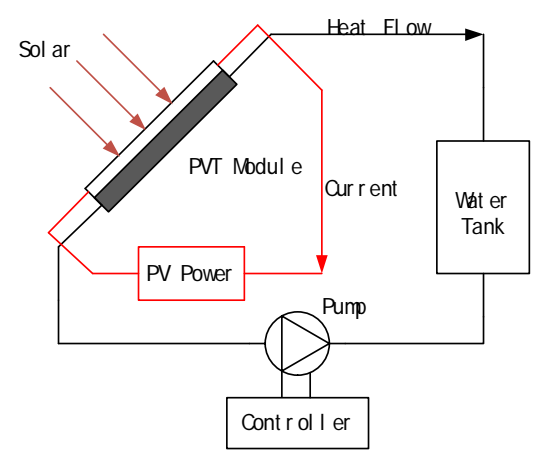

Fig. 2 PV / T system diagram

At present, the research on PV/T system is mostly carried out. The main types of PV/ T system and their research are discussed in [3-6]. The heat transfer performance and thermal efficiency of PV/T modules are compared. Literature [7-8] discussed the PV/T system and the combination with traditional heat pump composed of solar heat pump system, and compared the different solar thermal pump system thermal efficiency and stability of providing hot water. The relative literatures show that the PV/T system can improve the electrical efficiency and thermal efficiency in different degrees. However, the PV/T system running is mostly in extensive style, and little literature shows the PV/T system with the largest and the best use of solar energy for further energy saving. This paper aims at PV/T system for maximum and optimal use of solar energy.

In the PV/T system, the temperature control of $\mathrm{PV} / \mathrm{T}$ components is an important part of improving the efficiency of power generation and thermal efficiency. Only by controlling the temperature change of PV/T components can we carry out optimal control and realize the goal of maximum optimal use of solar energy. In this paper, a PV/T component temperature prediction method based on RBF neural network is proposed, which is beneficial to the optimal control of $\mathrm{PV} / \mathrm{T}$ system, for PV/T system is a big inertia system, temperature control effect needs a period of time. Due to the complexity of optimization control, this paper focuses on the PV/T system under sunny weather.

\section{PV/T component temperature under sunny weather}

In order to study the change of $\mathrm{PV} / \mathrm{T}$ component temperature, the $\mathrm{PV} / \mathrm{T}$ component temperature data from 2016-9-1 to 2016-10-1 in Nanning area of Guangxi were collected by PV/T monitoring system, Fig. 3 shows the PV/T components and PV components temperature changes under sunny weather.

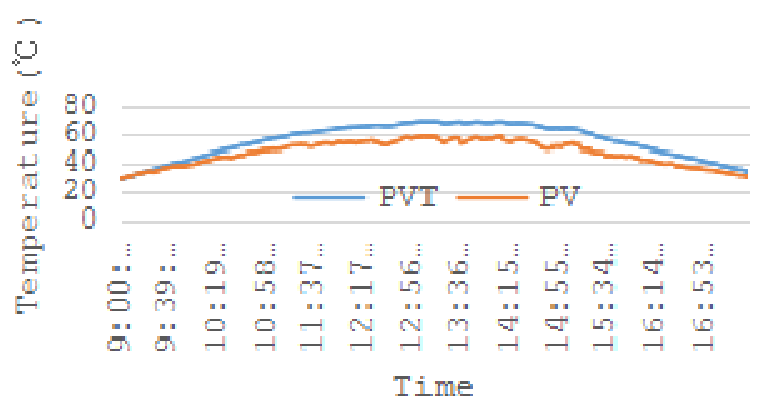

Fig. 3 Sunny temperature data

From the temperature curve of Fig.3, we can see that the PV/T component temperature is higher than the PV module temperature, the maximum difference of about $20{ }^{\circ} \mathrm{C}$, indicating that 
$\mathrm{PV} / \mathrm{T}$ components have a good insulation effect, which can improve PV/T system thermal efficiency. In addition, the PV/T component temperature changes flat, unless the external weather changing relatively large and for a longer time, will cause fluctuations, indicating that PV/T components have a strong temperature anti-interference capability to improve the prediction accuracy of PV/T components.

\section{PV/T component temperature prediction method selection}

The temperature curve of the PV/T component in sunny weather shows that the temperature and time are a nonlinear relationship. RBF neural network, as a newer artificial neural network model, can approximate any nonlinearity with arbitrary precision, with the only best approximation characteristics, and the structure is simple, training speed [9]. In the literature [10], the prediction accuracy of RBF neural network and BP neural network in predicting complex nonlinear relationship is analyzed from three aspects: prediction residual, relative error and prediction accuracy on mutation value. The results show that RBF neural network has the obvious prediction advantage. Therefore, the RBF neural network is used to analyze the PV/T component temperature.

\section{RBF neural network}

In the RBF network structure, there are three layers of structure, namely, input layer, hidden layer, output layer, and each node connected with the input layer in hidden layer has its own center as shown in Fig. 4. The role of the hidden layer node is to non-linear mapping of the input vector, and then to linear mapping of the output layer [11]. Each node of the hidden layer calculates the distance between input vector and its center, and then applies it to the excitation function to get the output of the hidden layer node. The output of the output node is the linear combination of the output of the hidden layer node. In this way, the hidden layer is obtained by a non-linear mapping of the input vector, and then through a linear mapping to the output. Excitation functions in the hidden layer are the same, the only difference is their center. The following is a commonly used one-dimensional radial basis function:

$$
f(x)=\exp \left(-\frac{\|x-c\|^{2}}{2 \delta^{2}}\right)
$$

Determining this function requires two parameters: the center $c$ and the variance $\delta$.

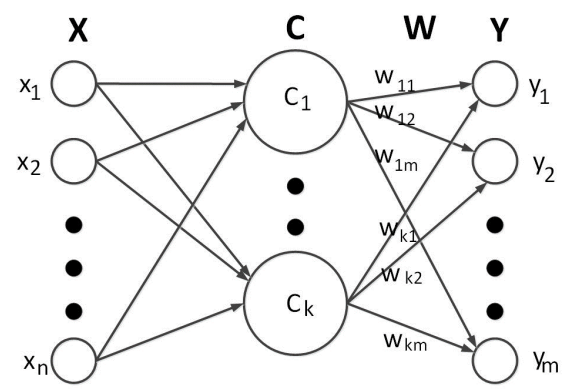

Fig. 4 RBF neural network structure

\section{Temperature prediction of PV/T component based on RBF neural network}

Data and data normalization. The laboratory PV/T monitoring system collects a set of data every minute, including the PV component, and the PV/T component temperature data. Based on the PV/T component temperature data from 2016-9-1 to 2016-10-1, the newrb function is used to 
predict temperature by MATLAB2015b software [12-14], and the validity and feasibility of the proposed method are analyzed by comparison.

According to the mathematical characteristics of the radial basis function, if the distance of the number to the radial basis function center is smaller than a certain value, can output more clearly. This article uses the "scale map method" for data normalization [15].

Experimental results and analysis. First select the PV/T component temperature data from 2016-9-1 to 2016-9-15 in sunny day, and select a group of data from 9:00 to 17:00 every 3 minutes as network training data to establish data model. And then through the data model, predict the PV/T component temperature data from 9:00 to 17:00 every 15 minutes of 2016-9-10 which is randomly selected one sunny day from 2016-9-1 to 2016-9-15 The prediction result and the prediction error are shown in Fig. 5 and Fig. 6, respectively.

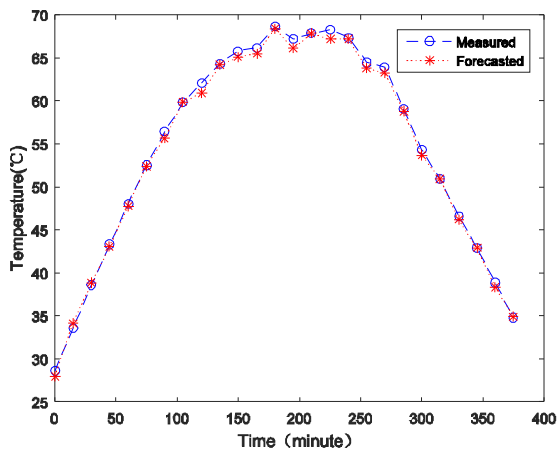

Fig. 5 Forecast results of 2016-9-10

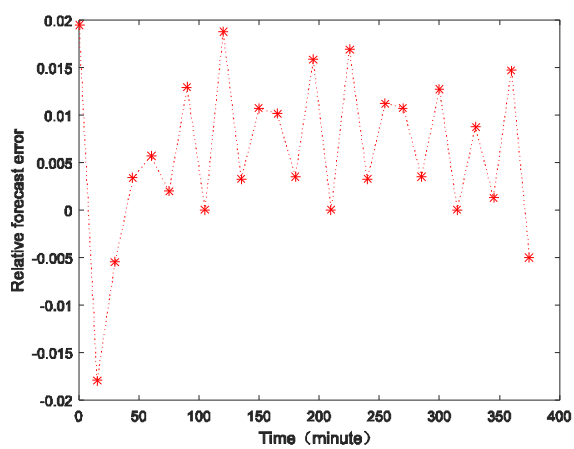

Fig. 6 Relative forecast error of 2016-9-10

The results show that the PV/T component temperature can be well predicted by RBF neural network, and the error between prediction and measured value is small and the maximum relative error is $2 \%$.

In order to further analyze the predictive performance of RBF neural network, as the same way, the author predict the PV/T component temperature data of 2016-9-20 which is randomly selected one sunny day from 2016-9-15 to 2016-10-1. The prediction results and the prediction errors are shown in Fig. 7 and Fig. 8, respectively.

It can be seen that the prediction error of $\mathrm{PV} / \mathrm{T}$ component temperature is relatively large at about 9:00 and 17:00, and the maximum relative error is about $8 \%$. When the illumination is sufficient at noon, the error is relatively small, because although it is sunny weather, the daily ambient temperature, wind speed, humidity, etc. is different, especially at 9:00 and 17:00, difference is obvious, resulting in PV/T component temperature has a certain extent fluctuation, but Fig. 8 shows most of the time the relative prediction error is less than $2 \%$, and the RBF neural network has good tracking ability for component temperature fluctuation.

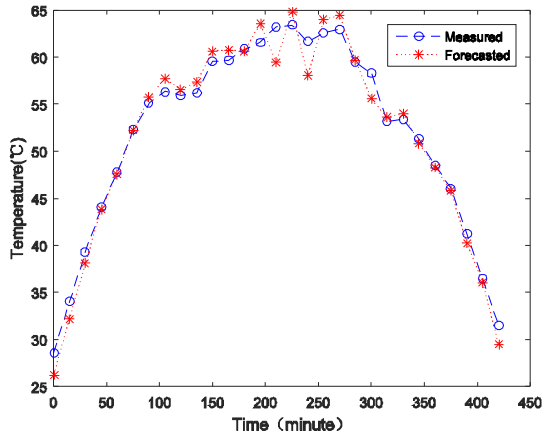

Fig.7 Forecast results of 2016-9-20

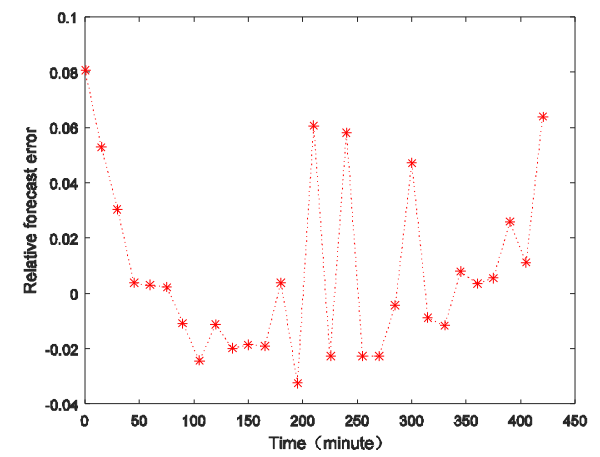

Fig.8 Relative Predictive Errors of 2016-9-20 


\section{Conclusions}

Based on the current PV/T system running in extensive style, this paper proposes the optimization study of PV/T system to achieve the maximum and best use of solar energy. Based on the analysis of the temperature of PV/T components in sunny weather, this paper proposes a prediction method based on RBF neural network, and draws the following conclusions:

1)According $\mathrm{PV} / \mathrm{T}$ component temperature prediction to adjust water flow rate, can provide technical support to the maximum and best use of solar energy.

2)Due to the influence of weather factors, the temperature of PV/T components fluctuates on different days. Although RBF neural network has strong fluctuation tracking ability, but the prediction accuracy is reduced, when the weather information changes greatly, the prediction method needs to further improve.

\section{Acknowledgements}

This work was financially supported by the Guangxi Natural Science Foundation (2014GXNSFAA118372), and the Scientific Research Fund of Guangxi Education Department ( 2013YB015).

\section{References}

[1] Xinbo Ruan, Zhiling Liao.[J]. Journal of Solar Energy.2009.30(4).430-435.

[2] Information on http://www.trinasolar.com/cn/.

[3] Jun Zhao, Na Qin. [J]. Journal of Solar Energy, 2009.30 (3). 327-331.

[4] G. Evola, L. Marletta. [J]. Solar Energy 107 (2014) 12-25.

[5] Baoqun Wang, Qiang Yao, Qiang Song . [J]. Journal of Solar Energy, 2009 (02): 193-200.

[6] Ahmad Fudholi, Kamaruzzaman Sopian. [J]. Energy Conversion and Management 78 (2014) 641-651.

[7] Peng Liu, Xin Guan, Zhijun Mu. [J]. Progress in Chemical Industry, 2009, 28 (S1): 328-333.

[8] Jie Jie, Qing-yin Jiang. [J]. Pattern Recognition and Artificial Intelligence, 2002, 15 (3): 310-316.

[9] Calise F, Dentice d'Accadia M, Vanoli L. [J]. Energy Convers Manage.52(2011).1562-73.

[10] Guangming Li, Zuming Liu, Jingtian Li. [J]. Proceeding of the CSEE, 2013,33 (17): 83-89.

[11] Ruidong Xu, Yi Dai, Xiaoyan Sun. [J]. Industrial automation, 2012, 38 (7): 59-63.

[12] Jinjun Pan, Yanbo Shen, Zeqiang Bian. [J]. Journal of Applied Meteorology, 2014, 25 (2): 150-157.

[13] Program, Zhiping Tan. [J]. Internet of Things Technology, 2013 (11): 32-33.

[14] Dong-dong Lu, Pu-yan ZHENG, Yan-zhou Yuan. [J]. Silicon Valley, 2014 (15): 43-44.

[15] Tao Hai, Lu Wang, Chunhua Chen.[J]. Journal of Guangxi University (Natural Science Edition), 2016,41 (5): 1508-1513. 